\title{
Procesos, dinámicas y tendencias de las políticas públicas nacionalesenrelación al desarrollo territorial y la gestión local en
} Argentina (2003-2017)

\author{
Processes, dynamics and trends of national public policies in \\ relation to territorial development and local management in
} Argentina (2003-2017)

ARTÍCULO

\begin{abstract}
Alejandro Casalis
Facultad Latinoamericana de Ciencias Sociales, Argentina.

Contacto: afcasalis@yahoo.com.ar
\end{abstract}

Recibido: mayo de 2017

Aceptado: agosto de 2017

\begin{abstract}
Resumen
En los últimos años cobra interés la reflexión sobre los procesos y las dinámicas que generan las políticas públicas nacionales sobre la gestión local y los territorios. Consideramos que en el período 2003-2015 asistimos a un proceso de recentralización de las políticas públicas nacionales destinadas al desarrollo territorial que introdujeron transformaciones en la agenda municipal, en el entramado de actores y en las posibilidades de desarrollo de los territorios. El cambio de gobierno a partir de diciembre de 2015 abre interrogantes sobre dicho proceso. ¿Qué características adoptan las políticas nacionales en relación al desarrollo territorial?, ¿qué consecuencias tiene sobre la agenda local? y ¿cuál es el entramado de actores que promueve? El artículo busca indagar en los procesos, dinámicas y tendencias más recientes de las políticas públicas nacionales, en relación al desarrollo territorial y la gestión local en Argentina, en el período 2003-2017.
\end{abstract}

Palabras claves: recentralización; descentralización; gestión local; políticas públicas.

\begin{abstract}
In recent years, reflection on the processes and dynamics generated by national public policies on local management and territories is of interest. We consider that in 2003-2015 we witnessed a recentralization process of national public policies aimed at territorial development that introduced transformations in the municipal agenda, in the framework of actors and in the development possibilities of the territories. The change of government as of December 2015 opens questions about this process. What are the characteristics of national policies in relation to territorial development? What consequences does it have on the local agenda? And what is the stakeholder structure it promotes? The paper seeks to investigate the processes, dynamics and more recent trends of national public policies in relation to territorial development and local management in Argentina in the period 2003-2017.
\end{abstract}

Keywords: recentralization; decentralization; local management; public policies. 


\section{Introducción}

En los últimos años cobra interés la reflexión sobre los procesos y las dinámicas que generan las políticas públicas nacionales sobre la gestión local y los territorios. La crisis del 2001 puso de manifiesto la pérdida de la capacidad del Estado para garantizar ciertos niveles de integración social y la resolución de conflictos a nivel nacional y local. En el período 2003-2015, se asiste a un proceso de recentralización (Vilas, 2010; De Piero, 2013; García Delgado, 2013; Cravacuore, 2014) de las políticas públicas nacionales destinadas al desarrollo territorial que introdujeron transformaciones en la agenda municipal, en el entramado de actores y en las posibilidades de desarrollo de los territorios.

El artículo tiene como objetivo indagar en las dinámicas y las tendencias de las políticas públicas nacionales en relación al desarrollo territorial y la gestión local. Para esto, se establece como hipótesis general que a partir de diciembre de 2015 , con el triunfo del gobierno de Cambiemos se produce una modificación en el rol del Estado y en su concepción de desarrollo que se plasma en un principio de reversión del proceso de recentralización del Estado producido durante el período 2003-2015 y una transferencia de funciones "de hecho" a nivel local sin la correspondiente dotación de recursos y capacidades. Esto tiene consecuencia para la gestión local y el desarrollo territorial y abre interrogantes sobre la evolución del sistema municipal argentino.

En este marco, las siguientes preguntas guían la investigación: ¿Cuáles son las dinámicas y las tendencias más significativas de este proceso? ¿Cómo operan y conviven procesos de recentralización con mecanismos de descentralización? ¿Cuáles son los aspectos más significativos que muestran estas modalidades? Para ello, en primer lugar, se realiza una breve caracterización sobre el proceso de descentralización en el marco de las Reformas del Estado a partir de 1990 que dio lugar al cambio en el modelo de gestión municipal y la adopción de nuevas competencias y funciones que éstos asumen. En segundo lugar, se analiza el proceso de recentralización del Estado en el período 2003-2015 y el correlato que tuvo en la gestión local y el desarrollo territorial. En tercer lugar, se indaga en las tendencias que se avizoran para la agenda local y el desarrollo territorial a partir del triunfo de Cambiemos en diciembre de 2015. Finalmente, en términos metodológicos se realiza un análisis cualitativo de los datos recogidos por medio de entrevistas en profundidad realizadas a funcionarios nacionales y locales y a especialistas en la temática, como así también el procesamiento de la información proveniente de documentos institucionales de políticas públicas y de fuentes periodísticas.

\section{1-Descentralización e implicancias a nivel local}

En el marco del proceso de Reforma del Estado iniciado a partir de 1990, la 
descentralización de competencias a niveles inferiores de gobierno (en primer lugar hacia las provincias y de éstas, hacia los municipios y comunas) supuso un cambio en el rol y en la relación de los gobiernos locales y con la comunidad. Al tradicional modelo de gestión municipal, los gobiernos locales incorporan nuevas competencias y funciones, y asume un rol más activo para responder a las demandas de desarrollo productivo, infraestructura, trabajo, vivienda, educación, política social, ambiente, participación social, entre otros (García Delgado, 1996; Cravacuore, 2007).

Las políticas de descentralización no sólo suponen el traslado de competencias y funciones sino también el pasaje de poder y atribuciones desde los gobiernos nacionales hacia los gobiernos locales. Pírez y Herzer (1988) señalan que "la descentralización constituye un proceso en virtud del cual ámbitos territoriales menores del Estado amplían su poder efectivo tomando funciones y recursos que estaban siendo ejercidos, de manera concentrada, por ámbitos superiores" (p. 209). Blutman (1999) define a la descentralización como "el mecanismo por el cual un conjunto de funciones y decisiones concentradas a nivel central se transfieren a unidades que tienen potestades sobre una jurisdicción territorial menor contenida en la anterior" (pp. 178-179). Cao y Vaca (2006) señalan que la descentralización implica como condición incorporar una relación directa con la ciudadanía para que haya descentralización efectiva, debido a que uno de los criterios explícitos es hacer más próxima la "gestión" a la satisfacción de necesidad y demandas de la ciudadanía.

El nuevo rol que asumen los municipios a partir del proceso de descentralización se sustentó en los supuestos de búsqueda de legitimidad (mayor cercanía a las demandas de la ciudadanía a nivel local) y racionalidad (mayor eficiencia y efectividad) (Chiara, 2012), y mayor control de la sociedad civil en los asuntos públicos (González Bombal y Villar, 2005); apelando al principio de subsidiariedad debido a la mayor proximidad del municipio con las demandas de la población (De Piero, 2010).

En términos procedimentales, se identifican tres tipos de descentralización: a) la fiscal, que implica transferir recursos; b) la política, que supone redistribuir poder hacia los gobiernos locales y hacia la comunidad; c) la institucional, que implica delegar competencias a nivel local. En el caso de Argentina los resultados obtenidos distan de coincidir con los esperados o promovidos. Una evaluación crítica del proceso de descentralización indica que lejos de promover mayor autonomía y dotar de recursos y capacidades a los gobiernos locales, éste estuvo caracterizado por la preeminencia de criterios fiscales y por la transferencias de responsabilidades a los niveles subnacionales sin la adecuada dotación de recursos. Tampoco ha mejorado significativamente la eficiencia, eficacia y la calidad de la prestación de los sistemas públicos subnacionales, ni ha generado mayor nivel de control y de participación ciudadana. ${ }^{1}$

\footnotetext{
1 Para un análisis exhaustivo del proceso de descentralización puede consultarse Borja (2007), Arroyo (2003), Cao y Vaca (2007), Smulovitz, y Clemente (2004), entre otros.
} 
No obstante los problemas antes señalados, podemos considerar que el proceso de descentralización contribuye a explicar -junto a otros ${ }^{2}$ - los cambios introducidos en el modelo de gestión y el fenómeno de la innovación de los gobiernos locales a partir de mediados de la década de 1990 (Iturburu, 2007; Cravacuore, llari y Villar, 2002). Como ya señalamos, esto dio lugar a cambios significativos en la agenda local y explica un mayor activismo de los gobiernos locales en relación con la planificación, la promoción del desarrollo, la innovación en la gestión y la pretensión de constituirse en un actor con cierta agenda propia -como se verifica durante la década de 1990 y en la mayor parte de la siguiente donde la temática municipal mostró mayor vitalidad tanto en el ámbito académico como de gestión- y por momentos intención de instalar temas de interés en el debate nacional. En ese sentido, el proceso de descentralización contribuyó a revalorizar la temática local. Se amplió la agenda local, se profesionalizó la gestión municipal, se generaron políticas nacionales para el fortalecimiento de la gestión local y aumentó la producción académica y el vínculo con las universidades, dando cuenta de este proceso de crecimiento. ${ }^{3}$ La contracara de este proceso fue la "municipalización de la crisis" (Arroyo, 2003) y la puesta en evidencia de las debilidades de los gobiernos locales en cuanto a las capacidades de gestión.

\section{2- El proceso de recentralización del Estado en el período posneoliberal y la implementación de políticas públicas}

La crisis del Estado que se suscitó a fines de la década del noventa y a principios de la siguiente puso de manifiesto dos cuestionamientos: el primero en relación con su misión y rol en la sociedad y el segundo vinculado a su capacidad de intervención (De Piero, 2013). A partir de 2003 esto implicó un cambio en relación a su rol en la implementación de políticas públicas y un cuestionamiento al proceso de descentralización que había guiado las Reformas del Estado y las relaciones interjuridiccionales durante la década anterior. El Estado recupera márgenes de autonomía política y da lugar al inicio de un proceso de recentralización de su accionar frente a los agentes económicos y a los actores institucionales y de la sociedad civil (Vilas, 2010). Como señala De Piero (2013):

luego de un período caracterizado por la inestabilidad política, (...) la década ya concluida logró reposicionar a la política, como la arena propia del debate y el espacio capaz de orientar al conjunto de la sociedad. No se ausentaron los conflictos, sino que estos se desplazaron notablemente de una lógica de libre concurrencia, de

\footnotetext{
2 Otros fueron los procesos de reformas constitucionales a nivel provincial iniciados en la década de 1980 que introdujeron la cuestión de la autonomía municipal y los procesos de modernización y Reforma del Estado, como los más significativos.

${ }^{3}$ En el plano académico se destaca la incorporación de la temática dentro de las carreras de Ciencia Política y Administración Pública, la inclusión dentro de reuniones científicas (CLAD, SAAP, entre otros) y la realización de Congresos y Seminarios específicos como el de RED MUNI en Argentina.
} 
competencia en términos de mercado, a la centralidad estatal como actor clave en el complejo entramado económico y social, dotado de mayores niveles de autonomía frente a la resolución de aquellos (p. 169).

Este proceso se hace más evidente a partir del año 2007, una vez reconstituida la legitimidad política y la gobernabilidad, recuperada la economía y encauzados los principales reclamos laborales y sociales (García Delgado y Nosetto, 2006). La recuperación de la centralidad del Estado en la asignación de recursos y en la implementación de políticas públicas se observa en la mayoría de las áreas de gestión -políticas de infraestructura, industriales, empleo, educación, sociales, de desarrollo científico-tecnológico y territorial (García Delgado y Peirano, 2011)-, junto con la reinstalación en la agenda pública del debate sobre el desarrollo, tanto como orientación teórica así como directriz programática de las políticas públicas (Ferrer, 2011; Zurbriggen, 2008).

La recentralización de la dinámica estatal alinea orientación política con la planificación del desarrollo y políticas pública. Dicho proceso se puede observar, por ejemplo, en la política económica con el sostenimiento del mercado interno; en la política laboral con la reinstitucionalización del mercado de trabajo, la creación de las Oficinas de Empleo Municipal y en los Programas con despliegue territorial de Capacitación y Promoción del Empleo y del Autoempleo del Ministerio de Trabajo, Empleo y Seguridad Social de la Nación (en adelante MTEySS) (Casalis, 2013); en la política socioproductiva como el Plan de Desarrollo Local y Economía Social "Manos a la Obra" (Casalis y Altschuler, 2006; Casalis, 2009) y luego la creación del Plan Argentina Trabaja del Ministerio de Desarrollo Social de la Nación (en adelante MDS); en las políticas de infraestructura a nivel local del Ministerio de Planificación Federal; Inversión Pública y Servicios de la Nación; en la Asignación Universal por Hijo (en adelante AUH), las políticas de ampliación de las jubilaciones y el Plan PROCREAR de la Administración Nacional de la Seguridad Social (en adelante ANSES); y en los programas del Instituto Nacional de Tecnología Industrial (INTI) y del Instituto Nacional de Tecnología Agropecuaria (INTA), entre otros. De este modo, se comienza a consolidar un proceso recentralizador de la gestión estatal y de las relaciones interjuridiccionales en relación a la implementación de las políticas públicas, ${ }^{4}$ junto a un proceso de recentralización de los recursos por parte del Estado nacional. En ese sentido, tomando las tres relaciones que el Estado desarrolla con la sociedad: funcionales, materiales y de dominación (Oszlak, 1997) podemos considerar que en parte los logros de este proceso se vinculan con el despliegue de relaciones funcionales y materiales puesto que el Estado logra abordar problemas -en particular los de financiamiento e implementación de políticas que demandan conjuntamente articular soporte tecnológico, logística y despliegue territorial-

\footnotetext{
${ }^{4}$ También se recupera una dimensión de lo federal dentro de la planificación que se plasma priorizar regiones históricamente postergadas, aunque sin que esto se corresponda con un cambio cualitativo en los niveles de desigualdad regional.
} 
que no pueden emprender las provincias y los municipios por las debilidades en la capacidad de gestión o presupuesto (Mangas, Lopéz Accotto, Martínez, 2016; MECON, 2012).

No obstante, la ocurrencia de un proceso de recentralización no significa que el Estado dé marcha atrás a las tendencias descentralizadoras tan características del período anterior, sino que la descentralización adquiere, partir de entonces, un nuevo rol y sentido. Las políticas de descentralización pierden la centralidad y jerarquía que habían adquirido durante el modelo neoliberal para adoptar un criterio operativo e instrumental en la implementación de las políticas públicas a nivel provincial y local. Algunas evidencias y dinámicas que ponen de manifiesto la recuperación de la centralidad del Estado nacional en el diseño, la implementación y el financiamiento de las políticas públicas en el plano local son: i) el incremento de la presencia territorial del gobierno nacional, por ejemplo mediante agencias y dependencias de ministerios nacionales a nivel provincial y local; ii) el diseño e implementación las políticas nacionales para el desarrollo territorial; iii) centralización en los recursos, aunque mayor transferencia en términos absolutos para inversión y gasto; iv) alta la dependencia -que se mantiene del período anterior- al financiamiento nacional para la implementación de políticas a nivel local; v) la implementación directa de políticas públicas a nivel local "saltando" a las provincias como articuladores en la relación nación-municipios.

La recentralización de la gestión genera mayor presencia del Estado a nivel territorial y modifica relaciones de poder. La acción recentralizadora del Estado nacional, a través del ejercicio de su poder territorialmente (Mann, 2007) desplaza a unos actores territoriales, visibiliza a otros, y pone de manifiesto los conflictos existentes en el territorio. Las consecuencias de este proceso son diversas y tienen impacto sobre la agenda local. Si bien con esa nueva modalidad de intervención el Estado no da respuestas a todos los temas prioritarios que forman parte de la agenda local, ${ }^{5}$ la redefine, incluso en aquellos municipios que habían procurado mantener cierta autonomía en la definición de la propia agenda, con el correlato de dar lugar a la constitución de una tendencia lenta pero persistente de redirigir, acotar y/o condicionar la agenda local a las pautas y los lineamientos establecidos por las políticas públicas nacionales (Cravacuore, 2014).

Asimismo, se mantiene y profundiza una tendencia de los gobiernos locales a definir su agenda en relación a la "oferta" de políticas y fuentes de financiamiento que tienen origen en otros niveles jurisdiccionales. Los gobiernos locales paulatinamente van asumiendo el rol de implementador de políticas diseñadas definidas desde el nivel nacional. Dicha tendencia puede observarse en el caso del Plan Argentina Trabaja, Oficinas Locales de Empleo, el Programa Mejoramiento de Barrios (PROMEBA) y urbanización de "villas de emergencia",

\footnotetext{
${ }^{5}$ Según Cravacuore (2008) la agenda local es un indicador para entender las prioridades de gestión y las capacidades institucionales de los gobiernos locales. El autor elabora una tipología de gobiernos locales en Argentina según el tipo de agenda que desarrollan y divide a los gobiernos locales en tres grandes grupos: a) los que tienen capacidad de elaborar y ejecutar su agenda; b) los que se limitan a ejecutar las políticas nacional y provinciales sin capacidad de formular sus propias agendas; y c) los que ejecutan una agenda tradicional.
} 
en algunos casos producción de suelo urbano (PROCREAR), el desarrollo de infraestructura local (asfalto, mejoramiento del espacio público, financiamiento de la construcción y/o mejoramiento de la infraestructura municipal, la construcción de Salones de Usos Múltiples (SUM) en los barrios y centros vecinales, e incluso en algunos gobiernos locales el financiamiento de obras votadas en el presupuesto participativo con fondos nacionales y provinciales). En este punto queremos resaltar el riesgo de subordinar la agenda a financiamientos externos y a políticas nacionales que adquieren mayor significación, en lugar de hacer un aprovechamiento estratégico de la disponibilidad de recursos y programas nacionales existentes en función de una agenda propia.

La agenda socioproductiva y de infraestructura, en particular en los municipios de las áreas metropolitanas (conurbanos y capitales de provincias) y de las ciudades intermedias se reconfigura por el despliegue que el Estado nacional realiza en los territorios. Esto se manifiesta a partir de un proceso de territorialización del Estado (Perelmiter, 2012) mediante la presencia en el territorio de instituciones del nivel nacional con alta capacidad de gestión como es la ANSES (AUH, ampliación de las jubilaciones, otorgamiento de pensiones, políticas de capacitación y terminalidad educativa como el PROGRESAR y de vivienda como el PROCREAR), las Unidades de Gestión a nivel provincial y/o regional del MDS y del MTEySS, y de Planes y Programas como el Plan Argentina Trabaja, la construcción de los Centros de Integración Comunitaria (CIC) del MDS, los programas de capacitación laboral y la construcción de las Oficinas de Empleo Municipal del MTEySS o el Plan de Emergencia Habitacional y el Plan Federal de Vivienda del Ministerio de Planificación Federal, Inversión Pública y Servicios de la Nación, entre otros.

Por último, los nuevos roles que asume el Estado en cuanto a las relaciones funcionales y materiales (Oszlak, 1997) en el plano socioproductivo ponen de manifiesto la existencia de conflictos latentes por la distribución del ingreso, la participación social (Rofman, 2016); y el reconocimiento y la identidad de colectivos vulnerables, subordinados y/o postergados (De Piero, 2015). La traducción de esta visibilización tiene como correlato un reposicionamiento de algunos actores políticos, económicos y sociales de los territorios en términos políticos y de disputas de poder con los gobiernos locales. ${ }^{6}$

Desde mi punto de vista hay que evaluar los procesos de recentralización en relación a la contribución al desarrollo y al fortalecimiento de la gestión local. El proceso de recentralización con su manifestación a nivel local dio lugar a un conjunto de políticas que posiblemente los municipios, en la mayoría de los casos, no podrían abordar con los recursos y capacidades que disponen. El desafío para el fortalecimiento de la gestión local

\footnotetext{
${ }^{6}$ A modo de ejemplo, se podría mencionar algunos casos de municipios donde hubo ciertas resistencias a la implementación a nivel local de programas destinados a la conformación de cooperativas de trabajo donde participen movimientos sociales, la promoción de espacios que fortalezcan la organización comunitaria y el reconocimiento de derechos $(\mathrm{CIC})$, y algunas iniciativas vinculadas a la agricultura familiar.
} 
es aprovechar la agenda nacional para fortalecer la agenda local o teniendo capacidad para elaborar y ejecutar agenda local propia -en términos de Cravacuore (2008)- utilizar los recursos nacionales para "liberar" o disponer de los recursos locales propios que puedan usarse para sostener la agenda propia.

\section{3-Principio de reversión del proceso recentralizador con transferencia de funciones "de hecho" a nivel local a partir de fines de 2015}

El gobierno de Cambiemos asume a fines de 2015 con un discurso crítico respecto del proceso recentralizador del Estado producido durante el período 2003-2015. Las principales críticas remiten a la intromisión indebida del Estado sobre asuntos que serían materia principal del mercado, el exceso de regulación que afecta el proceso de acumulación del capital y un sobredimensionamiento del gasto público que genera inflación y es fuente de corrupción. Si el período anterior supuso un proceso de recentralización del Estado, el actual implica un principio de reversión del proceso recentralización ya que el Estado modifica su rol y relación con la sociedad. En ese sentido, reduce el ámbito de intervención estatal en la regulación económica y financiera, en el mercado de trabajo, en la planificación del desarrollo en beneficio del mercado. Además, se transfiere la responsabilidad por el bienestar a los individuos, las familias y la sociedad civil a partir de la desarticulación o la redefinición de los programas laborales, educativos, de salud y sociales; ${ }^{7}$ y la invocación al emprendedorismo y a la meritocracia como formas de movilidad social ascendente.

Asimismo, apela a un discurso a favor del federalismo e igualdad entre las provincias como condición para devolver a éstas los recursos genuinos que habían sido retenidos por el Estado nacional como forma de disciplinamiento político. La transferencia de recursos a los gobiernos provinciales y menores regulaciones laborales, comerciales, financieras y tributarias serían -en el discurso oficial- necesarias para la realización de inversiones ${ }^{8} \mathrm{e}$ impulsarían el desarrollo y la reducción de la pobreza. Sin embargo, en el primer año y medio de gobierno de Cambiemos no se verifica todavía la recuperación tan mentada. Por el contrario, el panorama provincial muestra problemas fiscales, mayor endeudamiento con dolarización y significativas diferencias en cuanto a la transferencia de recursos

\footnotetext{
${ }^{7}$ Los casos más significativos de redefinición son el Programa Argentina Trabajo y Ellas Hacen que originalmente se pensaba reducir a su mínima expresión -incluso eliminar- por considerar que eran fuente de clientelismo o ineficiencia, sin embargo, con posterioridad se ha optado por mantenerlos pero con menor difusión. En la actualidad, el Argentina Trabaja está vinculado a la Ley de Emergencia Social.

${ }^{8}$ Se observa una apuesta a aquellos sectores en los que Argentina tiene ventajas comparativas como el agropecuario, agroindustrial, de energías renovables, minero, el turismo, además del anuncio de obras de infraestructura. Es menor el estímulo puesto en los sectores industriales que están orientados al mercado interno, son menos competitivos internacionalmente y están asentados en los grandes centros urbanos.
} 
coparticipables a las provincias (Agosto, 2016); caída de la actividad (INDEC, 2017); deterioro de los indicadores económicos y sociales (INDEC, 2017; ODESA-UCA, 2017); lenta reactivación de la transferencia de fondos a los municipios para realizar o continuar con los programas y las obras programadas; quita de beneficios impositivos por ejemplo a los puertos patagónicos y apertura de las importaciones que afectan a las economías regionales y a los sectores industriales volcados al mercado interno, entre otros.

En el marco de las transformaciones antes mencionadas, ¿cómo afecta el cambio de orientación del Estado en relación a las políticas dirigidas al fortalecimiento de la gestión local y el desarrollo territorial? En lo político-institucional se plantea una menor centralización del Estado nacional en favor de las provincias como sujeto del desarrollo territorial. En este esquema lo municipal queda circunscripto a políticas de modernización de la gestión (por ejemplo el Programa Smart Cities), la elaboración de un ranking para la transferencia de recursos contra la evaluación de desempeño y la redefinición de algunas políticas socioproductivas del período anterior. ${ }^{9}$

A su vez, se introduce en la agenda el enfoque del emprendedorismo, se acentúa el de la responsabilidad social empresaria y adquieren relevancia las Organizaciones No Gubernamentales (ONG's) como interlocutores para abordar la cuestión social. No obstante, como demostración de cierto pragmatismo y búsqueda de mantener la gobernabilidad se mantienen los canales de negociación con los movimientos sociales y las estructuras territoriales. En este proceso de cambios y continuidades, pareciera debilitarse la centralidad que tuvo durante el gobierno anterior las estrategias de inclusión socioproductiva de los sectores vulnerables (trabajo autogestivo, agricultura familiar, economía social y solidaria, entre otros) mediante un enfoque de economía plural y de organización sociocomunitaria. La novedad de este proceso es un mayor reconocimiento e incipiente institucionalización de un sujeto como es el trabajador de la economía popular, pero sin un soporte de política pública que ponga en valor a la producción que realizan y lo fortalezca como sector. ${ }^{10} \mathrm{De}$ este modo, para dar respuesta a las demandas sociales conviven en el gobierno de Cambiemos nuevas enunciaciones discursivas y políticas públicas junto con políticas, actores y mecanismos de negociación que se mantienen del período anterior.

Por lo tanto, ¿cómo repercute esta dinámica en lo local? El principio de reversión del proceso de recentralización con transferencia de funciones "de hecho" no viene acompañado de un proceso de descentralización donde se transfieran competencias, recursos y capacidad para resolver conflictos desde los niveles subnacionales. Tampoco estamos en presencia de una descentralización impulsada explícitamente por el Estado

\footnotetext{
${ }^{9}$ Por ejemplo, las de empleo y capacitación del MTEySS y el Argentina Trabaja del MDS.

10 La Ley de Emergencia Social fija un salario complementario y la creación de un Consejo Nacional de la Economía Popular pero por el momento está limitado a un programa de transferencia de ingresos en el marco de los programas existentes, por ejemplo el Plan Argentina Trabaja.
} 
nacional -como fuera durante la Reforma del Estado realizada en la última década del siglo $\mathrm{XX}$ - sino que asistimos a lo que llamamos una transferencia "de hecho" de cuestiones que han dejado de ser total o principalmente abordadas por el Estado nacional, y que recaen sobre la agenda municipal sin una adecuada transferencia de recursos y fortalecimiento de las capacidades locales. En un contexto de cambio en el rol del Estado y su relación con la sociedad, la agenda municipal se sobrecarga por el deterioro de los indicadores económicos y sociales y las dificultades presupuestarias. La consecuencia de ello es una tendencia a un corrimiento $-\mathrm{y}$ en algunos casos un regreso- hacia una agenda local más tradicional (Cravacuore, 2007; Iturburu, 2007) y la ralentización o postergación de iniciativas vinculadas a la modernización de la gestión y al fortalecimiento de una agenda más comprometida con el desarrollo y la ampliación de la ciudadanía.

Por otra parte, en el actual escenario de reversión del proceso de recentralización no se da lugar a discutir la crisis del sistema municipal argentino. ${ }^{11}$ Dicho sistema está agotado desde hace años -esto ya se constataba en el período anterior- por falta de recursos, sobrecarga de responsabilidades, límites reales a la autonomía y déficits en las capacidades de gestión, sin embargo, esta problemática no parece formar parte de la agenda nacional. A su vez, y más grave aún, se mantiene la debilidad política del municipalismo argentino para instalar a nivel nacional y provincial una agenda estratégica sobre federalismo, coparticipación, desarrollo territorial, desconcentración productiva y demográfica que permite poner en cuestión los principales problemas que afectan al municipalismo en Argentina. En definitiva, las dinámicas y las tendencias que asume el principio de reversión del proceso de recentralización con transferencia de funciones de "hecho" abren una serie de interrogantes sobre el rol del municipio en el esquema político-institucional, su participación en la agenda del desarrollo y los aportes a la ampliación de la ciudadanía a nivel local.

\section{Conclusión}

En este artículo, se analizaron los procesos, las dinámicas y las tendencias que adquieren de las políticas públicas nacionales en relación al desarrollo territorial y la gestión local en Argentina en el período 2003-2017. Se señaló que entre 2003 y 2015 se produjo un proceso recentralizador de las políticas públicas y que a partir de diciembre de 2015 se dio paso a un principio de reversión del proceso de recentralización con transferencia de funciones "de hecho" a nivel local. Así, en primer lugar, se caracterizó modo introductorio el proceso de descentralización ocurrido durante la última década del siglo XX en el marco de las políticas de Reforma del Estado. A su vez, se indicó que el mismo introdujo cambios en

\footnotetext{
11 La referencia a la crisis del sistema municipal argentino fue formulada por Daniel Cravacuore
} en el I Congreso Nacional de Estado y Políticas Públicas (FLACSO-UMET) 2016. 
el modelo de gestión local y amplió la agenda municipal, pero al mismo tiempo se produjo sin las correspondientes transferencias de recursos y capacidades necesarias para atender al incremento de demandas y una mayor complejidad que asumió la agenda local.

En segundo lugar, dimos cuenta del proceso de recentralización del Estado en el período 2003-2015 y señalamos que éste generó cambios en la gestión local y en las políticas de desarrollo territorial. Asimismo, relegitimó la acción del Estado nacional y permitió una mejor y/o mayor prestación pública que de otro modo no podría haber sido satisfecha por los gobiernos locales con la dotación de capacidades y recursos propios o provenientes de la coparticipación. No obstante, también se dijo que el proceso de recentralización contribuyó a perpetuar y/o profundizar una serie de problemas de vieja data como: i) en el plano institucional no se fortalecieron a los gobiernos locales como nivel jurisdiccional ni se modificó la débil autonomía real de los municipios; ii) en el plano de la gestión no se mejoraron las capacidades sino que se reforzó la existencia de una agenda dependiente de los recursos nacionales; iii) en el plano político el Estado nacional se introduce con presencia efectiva en el territorio y con capacidad de visibilizar o constituir nuevos actores dando lugar a tensiones políticas a nivel local.

En tercer lugar, el período que se inició a partir de finales de 2015 mostró un principio de reversión del proceso de recentralización pero con transferencias de funciones "de hecho" a nivel local. Cuando referimos a transferencias de funciones "de hecho" a nivel local se quiso señalar que el mismo se produce sin un impulso descentralizador explícito desde el Estado nacional como fuera durante la última década del siglo XX sino a partir de una redefinición del rol en relación al mercado y a la sociedad. Asimismo, tampoco se pone en discusión el agotamiento del sistema municipal argentino. Esto en su conjunto abre una serie de interrogantes sobre la capacidad del Estado nacional, provincial y local para abordar los principales problemas del sistema municipal argentino en relación al desarrollo territorial, la gestión local y la mejora de las capacidades, una mayor autonomía económica local acorde al incremento de su participación en la ejecución del gasto público total, el abordaje de cuestiones de índole metropolitano, y el diseño de políticas públicas nacionales que contemplen las particularidades del sistema municipal argentino.

\section{Referencias bibliográficas}

Agosto, W., (2016). Provincias: déficit, deuda y nuevas reglas de responsabilidad fiscal. Programa de Política Fiscal, Análisis n 179. Buenos Aires. CIPPEC, Área de Desarrollo Económico.

Altschuler, B.; Casalis, A. (2006). Aportes del Desarrollo local y la Economía Social a una estrategia nacional de desarrollo. En: García Delgado, D.; Nosetto, L. (comps.). El Desarrollo 
en un contexto postneoliberal. Hacia una sociedad para todos (pp.71-117). Buenos Aires: Editorial Ciccus.

Arroyo, D. (2003). Los ejes centrales del Desarrollo local en Argentina. Buenos Aires: Jefatura de Gabinetes de Ministros.

Blutman, G. (1999). Modelos políticos de descentralización municipal. En: Blutman, G. (comp.). Investigación sobre Reforma del Estado, Municipios y Universidad. Buenos Aires: CIAP-IIA. Facultad de Ciencias Económicas. Universidad de Buenos Aires.

Borja, J. (2007). Descentralización. Una cuestión de Método. En: Acuña, C. (ed.). Lecturas sobre el Estado y las políticas públicas: Retomando el debate de ayer para fortalecer el actual (pp. 55-78). Buenos Aires: Jefatura de Gabinete de Ministros.

Cao, H.; Vaca, J. (2007). El fracaso del proceso descentralizador argentino. Una aproximación desde la crítica a sus supuestos conceptuales. Andamio. 4(7), 249-267.

Casalis, A. (2013). Análisis del Programa Oficinas de Empleo Municipal. Una mirada sobre la contribución de inserción laboral y a la gestión local de las políticas de empleo en Argentina (2005-2010). Revista Documentos y Aportes en Administración pública y gestión estatal 13, (21), 64-91.

Casalis, A. (2009). Programa Caprino y Desarrollo Local en el marco del Plan Manos a la Obra: el caso del municipio de Rosario Vera Peñaloza, La Rioja. Ponencia presentada en X Seminario de RED MUNI "Nuevo rol del Estado, nuevo rol de los Municipios". Universidad Nacional de La Matanza, 13 y 14 de agosto de 2009.

Chiara, M. (2012). Paradojas de la descentralización en salud en el Gran Buenos Aires, Argentina. Derroteros de la Construcción de política Sanitaria en un Escenario Local. Postdata 17(2), 199-236.

Cravacuore, D. (2014). La recentralización emergente en América Latina. En Fidel, C.; Villar, A. (Comps.). Miradas y Controversias del Desarrollo Territorial. Aproximación a un Enfoque Analítico (pp. 67-86). Buenos Aires: Ediciones del Centro Cultural de la Cooperación Universidad Nacional de Quilmes.

Cravacuore, D. (2007). Los municipios argentinos (1990-2005). En: Cravacuore, D.; Israel, R. (Comps.). Procesos políticos municipales comparados en Argentina y Chile (1990-2005) 
(pp. 25-49). Buenos Aires: Editorial de la Universidad Nacional de Quilmes-Universidad Autónoma de Chile.

Cravacuore, D.; Ilari, S.; Villar, A. (2002). El fenómeno de la innovación en los gobiernos locales. En: Cravacuore, D.; Ilari, S.; Villar, A. (2002). La articulación en la gestión municipal. Actores y políticas (pp. 12-18). Bernal: Universidad Nacional de Quilmes.

De Piero, S. (2015). El Estado como promotor de la participación comunitaria: Representaciones y prácticas en tensión en los Centros de Integración Comunitaria. Tesis de Doctorado. Doctorado en Ciencias Sociales y Humanidades. Universidad Nacional de Quilmes.

De Piero, S. (2013). Los años kirchneristas: recentralizar al Estado. Aportes para el Estado y la Administración gubernamental, № 30 (pp. 169-179). Buenos Aires: Asociación de Administradores Gubernamentales.

De Piero, S. (2010). Participación Ciudadana en Políticas Públicas. En: Recorridos del Tercer Sector. Informe de Desarrollo Humano de la Provincia de Buenos Aires (2009-2010) (pp. 64-7). La Plata: Fundación BAPRO.

Ferrer, A. (2011). Los problemas fundacionales y la densidad nacional. Revista de Ciencias Sociales, 3. (19).

García Delgado, D. (2013). Editorial. Estado y Políticas Públicas: hacia un nuevo paradigma. Revista Estado y Políticas Públicas 1, 14-18.

García Delgado, D.; Peirano, M. (Comps.) (2011). El modelo de desarrollo con inclusión social. La estrategia de mediano plazo. Buenos Aires: Ediciones CICCUS.

García Delgado, D.; Nosetto, L. (Comps.) (2006). El Desarrollo en un contexto postneoliberal. Hacia una sociedad para todos. Buenos Aires: Editorial CICCUS.

García Delgado, D. (1996). Nuevos escenarios locales. El cambio del modelo de gestión. En: García Delgado, D. (Comp.) (1996). Hacia un nuevo modelo de gestión local. Municipios y sociedad civil en Argentina. Buenos Aires: CBC-UBA.

González Bombal, I.; Villar, R. (2005). Organizaciones de la sociedad civil e incidencia en políticas públicas. Colección Gestión Social. Buenos Aires: Universidad de San Andrés. 
Herzer, H.; Pírez, P. (1988). El municipio, entre la descentralización y la crisis. En: Herzer, H.; Pírez, P. (Comps.) (1988). Gobierno de la ciudad y crisis en Argentina (pp. 217-223). Buenos Aires: Grupo Editor Latinoamericano.

INDEC (2017). INDEC Informa, Nº 5 . Recuperado de (13/06/2017).

http://www.indec.gob.ar/ftp/cuadros/publicaciones/indecinforma/indec_informa_05_17.pdf Iturburu, M. (2007). La modernización en los municipios argentinos. En Cravacuore, D.; Israel, R. (Comps.). Procesos políticos municipales comparados en Argentina y Chile (19902005). Buenos Aires: Editorial de la Universidad Nacional de Quilmes- Universidad Autónoma de Chile.

Mangas, M.; Lopéz Accotto, A.; Martínez, C. (2016). La situación fiscal de las ciudades argentinas. En: RIEM. 14, 151-184.

Mann, M. (2007). El poder autónomo del Estado: sus mecanismos, orígenes y resultados. En: Acuña, C. (Comp.) (2007). Lecturas sobre el Estado y las políticas públicas: Retomando el debate de ayer para fortalecer el actual (pp. 55-78). Buenos Aires: Jefatura de Gabinete de Ministros.

MECOM (2012). Desempeño Fiscal reciente de los Gobiernos Locales en Argentina. Dirección de Análisis de Endeudamiento Provincial y Finanzas Municipales. Dirección Nacional de Coordinación Fiscal con las Provincias. Ministerio de Economía y Finanzas Públicas de la República Argentina. Ponencia presentada en el XI Seminario de RedMuni. Universidad Nacional Arturo Jauretche.

ODESA-UCA (2017). Pobreza y desigualdad por ingresos en la Argentina urbana 20102016. Marzo 2017. Observatorio de la Deuda Social. Buenos Aires: Universidad Católica Argentina. Recuperado de (13/06/2017):

http://www.uca.edu.ar/uca/common/grupo68/files/2017-Observatorio-Informe-Pobreza-

Desigualdad-Por-Ingresos-2010-2016.pdf

Oszlak, O. (1997). Estado y Sociedad ¿Nuevas reglas del juego?. En: Revista Reforma y Democracia 9, 1-41.

Paramio, L. (2008). El regreso del Estado: entre el populismo y la regulación. En: Revista Reforma y Democracia 42. 
Perelmiter, L. (2012). Burocracia, pobreza y territorio. La política espacial de la asistencia en la Argentina reciente. Ponencia presentada en la VII Jornadas de Sociología de la Universidad Nacional de General Sarmiento. Los Polvorines, Buenos Aires. Recuperado de (22/05/2017): $\quad$ http://www.ungs.edu.ar/ms_ici/wp-content/uploads/2012/11/Perelmiterponencia-versi\%C3\%B3n-final.pdf

Rofman, A. (Comp.) (2016). Participación, políticas públicas y territorio: aportes para la construcción de una perspectiva integral. Los Polvorines: Universidad Nacional de General Sarmiento.

Smulovitz, C.; Clemente, A. (2004). Descentralización, sociedad civil y gobernabilidad democrática en Argentina. En: Clemente A.; Smulovitz C. (Comps.) (2004). Descentralización, políticas sociales y participación democrática en Argentina (pp. 39-89). Buenos Aires: IIED-AL.

Vilas, C. (2010). Después del neoliberalismo: Estado y procesos políticos en América Latina. Remedios de Escalada: Ediciones de la Universidad Nacional de Lanús.

Zurbriggen, C. (2008). La nueva agenda del Desarrollo ¿Dónde queda el Estado?. Nueva Sociedad 42. 